\title{
Assessment of value of calibrated lyophilised plasmas to determine International Sensitivity Index for coagulometers
}

\author{
K Clarke, D A Taberner, J M Thomson, J A Morris, L Poller
}

\begin{abstract}
An attempt was made to correct for the effects of coagulometers on the International Sensitivity Index (ISI) in a series of collaborative studies. Modified ISI were derived from the prothrombin time results with coagulometer systems using a range of calibrated plasmas. Two alternative approaches to correction of the ISI were evaluated. The first relied on the consensus orthogonal regression slopes of the prothrombin times for each coagulometer system plotted against the consensus manual results; the second depended on the local individual slope of the prothrombin times for each instrument. The two procedures were compared with the currently recommended method where International Normalised Ratios (INR) are derived from the manual ISI of the thromboplastin. The recommended method gave a significant bias from the manual results with most coagulometers. In contrast, the local correction procedure gave no significant biases, whereas the consensus method did so in a few instances. Both these correction procedures seem more reliable than the recommended method of INR derivation, but the local correction is more accurate and offers a more practical solution by allowing laboratories to determine their own corrected ISI on a range of calibrated plasmas.
\end{abstract}

For monitoring oral anticoagulant control most routine coagulation laboratories in the United Kingdom now use an automated technique for the determination of the prothrombin time (PT). Results are reported as International Normalised Ratios (INR). INR were designed to compensate for the different responsiveness (International Sensitivity Indices-ISI) of the various thromboplastin reagents. ${ }^{1}$ The ISI value of a thromboplastin, however, which, by definition is derived from the manual technique, is changed by most coagulometers. ${ }^{2-5}$

In this study an attempt has therefore been made to correct for the effects of automated instruments on the ISI. The relation between coagulometer and manual PT was determined by means of a range of lyophilised plasmas. Correction procedures for coagulometer effects were evaluated in four serial multicentre exercises.

Two alternative approaches to correction were adopted. One of these relied on the relation between the consensus instrument results for each brand of coagulometer and the consensus manual results. A consensus instrument specific ISI for each type of coagulometer using one brand of thromboplastin (system) was thus derived. The second procedure was based on the relation between the results with an individual local coagulometer and the consensus manual mean. The two correction procedures were based on cumulative data from the first three exercises. The reliability of both procedures was tested in the fourth exercise using lyophilised samples obtained from patients treated with coumarin, comparing percentage bias from the consensus manual INR.

\section{Methods}

One hundred and forty nine laboratories returned results from all four serial exercises. Of these, 66 laboratories performed a manual test and 81 an automated procedure in sufficient numbers for a system analysis. To simplify the study it was confined to users of one thromboplastin, Manchester Reagent. A range of artificially depleted lyophilised plasmas spanning the therapeutic interval of $2 \cdot 0$ to 4.5 INR was included in each exercise. Eight plasmas were issued in the first and nine in the subsequent surveys. In the last survey three lyophilised coumarin plasmas were also included to evaluate the two correction procedures.

\section{CORRECTION PROCEDURES}

Orthogonal regression slopes for the two correction procedures were obtained by plotting $\log$ PT with both coagulometer and manual results. Manual mean INR results were used as reference values to calculate percentage bias of the instruments.

The modified ISI for the coagulometer systems were derived by multiplication of the stated ISI value from the manual method of calibration with the value of the orthogonal regression slope. These slopes were obtained using two different procedures-that is, from: (a) the consensus slope for each system from the results of all 26 test plasmas issued in the first three surveys. Consensus mean normal prothrombin times (MNPT) were used to 
Table 1 Effect of coagulometers on ISI

\begin{tabular}{lllll}
\hline Method & $\begin{array}{l}\text { Consensus } \\
M N P T\end{array}$ & $\begin{array}{l}\text { Manual ISI } \\
\text { of MR } \\
\text { thromboplastin }\end{array}$ & $\begin{array}{l}\text { Consensus } \\
\text { slope }\end{array}$ & $\begin{array}{l}\text { Coagulometer } \\
\text { ISI }\end{array}$ \\
\hline ACL & 12.31 & 1.09 & 0.9452 & 1.0303 \\
Biomatic & 15.17 & 1.09 & 0.8702 & 0.9485 \\
Burkard (S and G) & 14.90 & 1.09 & 0.9572 & 1.0433 \\
Coag-a-mate & 13.74 & 1.09 & 0.9839 & 1.0725 \\
Fibrintimer & 14.30 & 1.09 & 0.9636 & 1.0503 \\
KC4/KC10 & 14.57 & 1.09 & 0.9098 & 0.9917 \\
\hline
\end{tabular}

derive prothrombin ratios for the INR calculation;

(b) the local slope, from each individual laboratory's results with all 26 test plasmas issued in the first three surveys. The local MNPT value was used to derive the prothrombin ratios for INR calculation.

The two above procedures were compared with the method currently recommended (c) where INR are derived from the prothrombin ratio obtained using the local MNPT and the manufacturer's stated ISI of the thromboplastin.

These three methods of INR derivation were then evaluated for each individual laboratory's results on the three coumarin plasmas included in the fourth exercise.

\section{STATISTICAL METHODS}

The bias for each reading was computed by subtracting the consensus manual INR results from the instrument INR values. These biases were then divided by the consensus manual INR results to give percentage biases. Ninety five per cent confidence intervals for the bias expected for a single instrument INR reading were calculated as described by Bland and Altman. ${ }^{6}$ The three methods of INR deriva-

Table 2 Mean percentage bias (95\% confidence interval) *

\begin{tabular}{|c|c|c|c|}
\hline Instrument & Plasma I & Plasma II & Plasma III \\
\hline Consensus Manual INR & $2 \cdot 60$ & 3.47 & $3 \cdot 76$ \\
\hline $\begin{array}{l}A C L \\
\text { Method a }(\mathbf{n}=8) \\
\text { Method b }(\mathbf{n}=2) \\
\text { Method c }(\mathbf{n}=6)\end{array}$ & $\begin{array}{l}3(-8,14) \\
1(-10,12) \\
6 \neq \ddagger(-5,17)\end{array}$ & $\begin{array}{c}8(-16,32) \\
3(-21,27) \\
13 \ddagger \ddagger(-11,37)\end{array}$ & $\begin{array}{r}-4(-27,29) \\
0(-23.23) \\
0(-23,23)\end{array}$ \\
\hline $\begin{array}{l}\text { Biomatic } \\
\text { Method a }(n=6) \\
\text { Method b }(n=4) \\
\text { Method c }(n=5)\end{array}$ & $\begin{array}{r}-10 \dagger \dagger(-21,1) \\
-2 \dagger \dagger(-13,9) \\
10 \neq \ddagger(-1,21)\end{array}$ & $\begin{array}{c}-12 \ddagger \ddagger(-24,0) \\
-5 \ddagger \ddagger(-17,7) \\
11 \ddagger \ddagger(-1,23)\end{array}$ & $\begin{array}{c}-7 \S(-33,19) \\
0 \dagger \dagger \S(-26,26) \\
20 \neq \ddagger(-6,46)\end{array}$ \\
\hline $\begin{array}{l}\text { Coag-a-mate }(\times 2 \times C) \\
\text { Method a }(n=18) \\
\text { Method } b(n=7) \\
\text { Method } c(n=16)\end{array}$ & $\begin{array}{r}2(-21,17) \\
-3(-22,16) \\
4(-15,23)\end{array}$ & $\begin{array}{l}8 \ddagger \ddagger(-14,30) \\
1(-21,23) \\
9 \ddagger \ddagger(-13,31)\end{array}$ & $\begin{array}{c}22 \dagger \ddagger \ddagger(-38,82) \\
6(-16,28) \\
12 \ddagger \ddagger(-10,34)\end{array}$ \\
\hline $\begin{array}{l}\text { Fibrintimer } \\
\quad \text { Method a }(n=9) \\
\text { Method b }(n=4) \\
\text { Method c }(n=6)\end{array}$ & $\begin{array}{r}0(-16,16) \\
-5(-21,11) \\
0(-16,16)\end{array}$ & $\begin{array}{r}5(-12,22) \\
-2(-19,15) \\
4(-13,21)\end{array}$ & $\begin{array}{r}6(-13,25) \\
-2(-21,17) \\
5(-14,24)\end{array}$ \\
\hline $\begin{array}{l}K C 4 / K C 10 \\
\text { Method a }(n=33) \\
\text { Method b }(n=6) \\
\text { Method c }(n=18)\end{array}$ & $\begin{array}{r}1 \S(-19,21) \\
-1 \S(-21,19) \\
12 \ddagger \ddagger(-8,32)\end{array}$ & $\begin{array}{r}3 \$(-17,23) \\
1 \$(-19,21) \\
19 \ddagger \ddagger(-1,39)\end{array}$ & $\begin{array}{r}4 \$(-19,27) \\
1 \$(-22,24) \\
19 \ddagger \ddagger(-4,42)\end{array}$ \\
\hline $\begin{array}{l}\text { Burkard ( } S \text { and } G) \\
\text { Method } \mathrm{a}(\mathrm{n}=7) \\
\text { Method } \mathrm{b}(\mathrm{n}=2) \\
\text { Method c }(\mathrm{n}=6)\end{array}$ & $\begin{array}{l}-2(-11,7) \\
-3(-12,6) \\
1 \neq(-8,10)\end{array}$ & $\begin{array}{c}-2(-24,20) \\
0 \$(-22,22) \\
4(-18,26)\end{array}$ & $\begin{array}{l}-1 \S(-16,14) \\
-1 \S(-16,14) \\
5(-10,20)\end{array}$ \\
\hline
\end{tabular}

$\star 95 \%$ confidence interval for a single reading or actual percentage bias. tmean included one extreme reading.

†tsignificantly smaller absolute absolute bias compared with method a.

tsignificantly smaller absolute bias compared with method b.

significantly smaller absolute bias compared with method $c$.

ftmean absolute bias significantly greater than zero. tion were compared using multiple regression methods for each system and each of the three coumarin plasmas separately. The significance of individual biases was assessed by examination of the regression coefficients and their standard errors.?

\section{Results}

Table 1 shows the effect of coagulometers on the stated ISI of Manchester Reagent. Consensus slopes and coagulometer modified ISI values are listed. The extent of the influence of the various coagulometer systems on the manual ISI value is shown.

The mean and confidence intervals of the percentage bias with each system with the three coumarin plasmas are shown in table 2 . Analysis of results with the three procedures for INR derivation ( $a, b$, and $c$ ) are given for the coumarin plasmas with each coagulometer system. The numbers included varied because some participants did not provide complete sets of data. The currently recommended method for INR derivation (method c) gave a bias from the manual results which was significant with most of the systems. In contrast, the local correction procedure gave no significant biases whereas the consensus method did so with the two systems. Overall, the local calibration method (b) gave the least bias which achieved significance with the Biomatic, KC, and Burkard instruments when compared with the conventional method (c).

\section{Discussion}

The value of a range of calibrated PT plasmas in characterising local or consensus performance with different coagulometer systems was assessed in an attempt to derive an INR correction procedure to compensate for the instrument effects.

The use of coagulometer systems may greatly affect INR values and result in considerable differences from the manual technique. This study was limited to a single thromboplastin because it has been shown that the thromboplastin and instrument effect on the PT could be identified separately. ${ }^{8}$ Furthermore, it was noted that there was an interaction between instrument and reagent, so that a system effect on the ISI needed to be quantified. It has been suggested that this can be achieved by a system calibration obtained from one instrument of a particular brand with one type of thromboplastin. ${ }^{5}$ Parallel testing with the coagulometer and manual technique using an international reference preparation was required for such a calibration. ${ }^{5}$ This single centre approach does not take into account the evidence that there is considerable variation in performance between instruments of the same model. ${ }^{349} \mathrm{~A}$ single instrument may therefore not be representative. Consensus calibration by a group of users of an individual system or the calibration of each individual coagulometer at the local level are methods which might overcome the above difficulties. Our attempt at modification of the manual ISI to correct for the effect of coagulometers has been based on these 
two approaches-that is, the use of a global correction for each brand of coagulometer derived from its consensus values from the results of lyophilised calibrated plasmas-or alternatively a local correction based on an individual laboratory's results with these plasmas with a single coagulometer. Both of these procedures seem to be better than the currently recommended method of INR derivation based on the manually derived ISI of the thromboplastin. Furthermore, the local correction based on results of the calibrated plasmas with individual coagulometers proved to be generally more reliable than the consensus approach. Of the two proposed correction methods, the local calibration procedure also offers an easier and more practical solution by allowing laboratories to determine their own corrected ISI based on a range of plasmas. These need to be reliably calibrated with prothrombin time values by the recommended manual technique.
We thank Manchester Comparative Reagents for financially supporting this study.

1 World Health Organisation. Expert Committee on Biological Standardisation. 33rd Report. WHO Technical Report Series. Geneva: WHO, 1983:1-105.

2 Poller L, Thomson JM, Taberner DA. Effect of automation on the prothrombin time test in NEQAS surveys. J Clin Pathol 1989;42:97-100.

3 Thomson JM, Taberner DA, Poller L. Automation and prothrombin time: a United Kingdom field study of two widely used coagulometers. J Clin Pathol 1990;43:679-84

4 Ray MJ, Smith IR. The dependence of the International Sensitivity Index on the coagulometer used to perform the prothrombin time. Thromb Haemostas 1990;63:424-9.

5 Poggio M, van den Besselaar AMHP, van der Velde EA Bertina RM. The effect of some instruments for prothrom-
, Thio bin time testing on the International Sensitivity Index (ISI) of two rabbit tissue thromboplastin reagents. Thromb Haemostas 1988;62:868-74.

6 Bland JM, Altman DG. Statistical methods for assessing agreement between two methods of clinical measurement. Lancet 1986:i:307-10.

7 Aitkin M, Anderson D, Francis B, Hinde J. Statistical modelling in GLIM. Oxford: Clarendon Press, 1989.

8 Evatt BL, Brogan D, Triplett DA, Koepke J, Waters MPH Effect of thromboplastin and instrumentation on the prothrombin time test. Clin Lab Haematol 1981;3:331-42.

9 Prothrombin time test. Clin Lab Haematol 1981;3:331-42. techniques for the prothrombin time test: results of techniques for the prothrombin time test: result
proficiency studies. Br J Haematol 1978;38:391-9. 